\title{
The impact of 18F-FDG PET/CT in the clinical management of patients with lymph node metastasis of unknown primary origin
}

Petya Nikolaeva NIKOLOVA ${ }^{1, *}$, Valeria Hristova HADZHIYSKA ${ }^{1}$, Kiril Blagoev MLADENOV ${ }^{1}$, Mihaela Georgieva ILCHEVA ${ }^{1}$, Stefani VENEVA ${ }^{1}$, Violeta Vasileva GRUDEVA², Svetla Emilova DINEVA ${ }^{3}$, Yavor Nikolov ASENOV ${ }^{4}$

${ }^{1}$ Clinic of Nuclear Medicine, University Hospital Alexandrovska, Sofia, Bulgaria; ${ }^{2}$ Department of Imaging Diagnostics, University Hospital St. Ekaterina, Sofia, Bulgaria; ${ }^{3}$ Clinic of Imaging Diagnostics, University Hospital Alexandrovska, Sofia, Bulgaria ${ }^{4}$ Clinic of Surgery, University Hospital Queen Giovanna, Sofia, Bulgaria

${ }^{*}$ Correspondence: petia.nn@abv.bg

Received March 15, 2020 / Accepted July 1, 2020

\begin{abstract}
The aim of this study was to evaluate the diagnostic performance and the utility of F-18-fluorodeoxyglucose (FDG) positron emission tomography-computed tomography (PET/CT) in the clinical management of patients presenting with lymph node metastasis of undefined primary origin (CUP). A total of 53 patients (34 males, 19 females) with a diagnosis of lymph node metastasis according to the histopathology and/or conventional imaging were enrolled in this retrospective study. Patients were divided into four groups according to the initial location of their metastasis - group 1, cervical lymph nodes $(n=39)$, group 2 , axillary lymph nodes $(n=6)$, group 3 , mediastinal lymph nodes $(n=2)$ and group 4 , abdominal and pelvic lymph nodes $(n=6)$. The site of a probable primary malignancy suggested by PET/CT was confirmed by biopsy/ further investigations or follow-up. 18F-FDG PET/CT accurately detected the primary carcinoma in 19 of 53 patients (36\%), with head and neck cancer and lung carcinoma being the most common primary locations. The PET-CT scan results were negative for primary site localization in $13 \%$ of patients (false-negative), while $45 \%$ had true negative results, and $6 \%$ displayed false-positive results. Additional distant metastatic foci were identified in 21 of all patients (40\%). The overall sensitivity, specificity, and accuracy rates of the study were identified as $73 \%, 89 \%$, and $81 \%$, respectively; in the group with cervical lymph node metastasis sensitivity $70 \%$ and specificity $84 \%$. To conclude, $18 \mathrm{~F}$-FDG PET/CT is a sensitive and selective procedure for detecting unknown primary tumors, especially in the clinical setting of cervical lymph node metastasis and its use should be encouraged earlier in the pre-treatment phase of CUP-patients, leading to higher detection of probable primary sites, guiding subsequent biopsy, and more accurate detection of distant metastases in a single examination.
\end{abstract}

Key words: cancer of unknown primary site, 18F-FDG PET/CT, lymph node metastasis

Cancer of unknown primary site (CUP) is a well-recognized clinical syndrome, characterized by early dissemination, clinical absence of the primary tumor, the unpredictability of metastatic pattern, and aggressive behavior. CUPs account for $3-5 \%$ of all malignancies, being the seventh to eighth most frequently occurring cancer in the world and the fourth most common cause of cancer-related death in both men and women. More than 50\% of CUP patients present with multiple sites of involvement, while the rest have a single site, most commonly in the liver, bones, lungs, or lymph nodes $[1,2]$. Those with primary detected lymph node involvement represent a specific group due to the fact that some of these subsets require specific treatment approaches and have the potential for an excellent outcome as squamous cell carcinoma involving cervical lymph nodes, women with adenocarcinoma involving only axillary lymph nodes, poorly differentiated carcinoma with midline distribution (extragonadal germ cell), isolated inguinal adenopathy (squamous carcinoma), and patients with a single, small, potentially resectable tumor. Identification and treatment of these patients are of paramount importance, considering that the median overall survival varies in the range of one year [3-6]. Therefore, avoiding endless diagnostic studies and taking a rapid decision about treatment options is crucial.

Unfortunately, the conventional imaging methods identify the site of primary in only $20-27 \%$ of cases [7]. Fluorodeoxyglucose (FDG) positron emission tomography/computed tomography (PET/CT) is a valuable modern imaging technique for patients with CUP, particularly in those with squamous cell cancer in cervical lymph nodes. However, 
the detection rate of CUP by 18 F-FDG PET varies in a large range, between $8 \%$ and $57 \%$ in the literature with an overall rate of about 39\% [8-10]. Despite the observed heterogeneity in terms of detection rate, some centers even recommend it as an initial screening investigation for CUP [11].

The aim of this study is to evaluate the impact of F-18-fluorodeoxyglucose positron emission tomography-computed tomography in patients presenting with metastatic lymph node involvement of undefined primary origin.

\section{Patients and methods}

This is a retrospective study of 53 patients with isolated lymph node involvement from unknown primary, who were referred for 18F-FDG PET/CT scan during the period 2012-2016. Forty-nine out of 53 patients were proved to have metastases histopathologically and only four patients had highly suspicious metastases by conventional imaging. However, prior to receiving the F-18 FDG PET/CT scan, the primary could not be confirmed using regular methods, including detailed physical examination, serum tumor marker test, and other imaging auxiliary examinations including chest X-ray, CT, magnetic resonance imaging (MRI), mammography (in females), cervical ultrasonography, and endoscopy. Exclusion criteria were the detection of a primary tumor by any other investigation tool. The included patients were divided into several groups according to the initial localization of metastatic foci-group 1 (cervical lymph nodes, $\mathrm{n}=39$ ), group 2 (axillary lymph nodes, $\mathrm{n}=6$ ), group 3 (mediastinal lymph nodes, $\mathrm{n}=2$ ), and group 4 (abdominal and pelvic lymph nodes, $\mathrm{n}=6$ ). The present study was approved by our institutional ethical review committee and all patients provided written informed consent prior to the PET/CT imaging.

Scan protocol. Whole body 18F-FDG PET/CT was performed by using an integrated PET/CT scanner (GE 600 Discovery, 16 Slice, Time of Flight). Patients were fasted for at least 6-8 hours before injection. Blood glucose level was below $10 \mathrm{mmol} / \mathrm{l}$ at the time of radiotracer injection. Sixty minutes after IV administration of $3-3.5 \mathrm{MBq} / \mathrm{kg}$ (270-370 MBq) 18F-FDG image acquisition commenced craniocaudally with the $\mathrm{CT}$ component performing by a multidetector CT scanner and the following parameters: auto mAs (50-120), $120 \mathrm{kV}, 2.5 \mathrm{~mm}$ slice thickness. The PET data were then collected in the reverse direction immediately after CT acquisition with a time of 2-4 minutes per bed position. All patients received an oral or intravenous contrast. When an intravenous contrast was administrated, the CT parameters were $50-340 \mathrm{mAs}$ and $120 \mathrm{kV}$. A total volume of $100 \mathrm{ml}$ of the IV contrast agent (Ultravist) was injected using a power injector at a flow rate of $2-3 \mathrm{ml} / \mathrm{s}$.

Image reconstruction and interpretation. Image readout was performed on an AW4.5 WorkStation (GE Healthcare), which allowed visualization of PET, CT, and fused sections in transverse, coronal, and sagittal planes. Maximum standard- ized uptake values were automatically generated by the software. The study was reviewed independently by two experienced nuclear medicine physicians. When an intravenous contrast was administrated, the images were evaluated along with a radiologist. PET/CT was considered positive when an increased FDG uptake indicative of a primary tumor was identified in the head and neck and/or other regions of the body, strongly correlating with the patient's medical record and pathological results. The goal was the ability of hybrid FDG PET/CT to detect the potentially primary tumor and/or all manifestations of distant lesions.

Data analysis and statistical evaluation. In the investigation of a primary tumor, detection of the primary malignancy site was considered to be true positive (TP) only when confirmed by histopathology and/or by clinical follow-up/ further investigations. If findings on the PET-CT scan did not turn out to be a primary site by histopathology, these were accepted as false positive (FP). If 18F-FDG PET/CT could not detect the primary tumor and it remained unknown, the result was considered true-negative (TN). When 18F-FDG PET/CT did not suggest any primary tumor but was diagnosed with conventional work-up or in the patient's follow-up, the result was considered as false-negative (FN).

Sensitivity, specificity rates, accuracy, positive predictive value (PPV), and negative predictive value (NPV) were calculated using standard statistical formulas with $95 \%$ confidence intervals: Sensitivity $=\mathrm{TP} /(\mathrm{TP}+\mathrm{FN})$, Specificity $=\mathrm{TN} /$ $(\mathrm{TN}+\mathrm{FP})$, Accuracy $=(\mathrm{TP}+\mathrm{TN}) /(\mathrm{TP}+\mathrm{FP}+\mathrm{TN}+\mathrm{FN}), \mathrm{PPV}=$ $\mathrm{TP} /(\mathrm{TP}+\mathrm{FP}), \mathrm{NPV}=\mathrm{TN} /(\mathrm{TN}+\mathrm{FN})$.

The receiver operating characteristic (ROC) analysis was executed using MedCalc Statistical Software version 19.3 (MedCalc Software Ltd, Ostend, Belgium). The ROC curve was plotted with sensitivity on the y axis against (1-specificity) on the axis. The AUC above 0.90 is considered to be a high diagnostic value. $0.70-0.90$ is a moderate diagnostic value and less than 0.70 is a poor diagnostic value.

\section{Results}

A total of 34 male patients (64\%) and 19 female patients (36\%) were included. Detailed clinicopathological data of the patients are shown in Table 1 . In 10 patients, 18F-FDG $\mathrm{PET} / \mathrm{CT}$ was used as a first-line imaging modality, while in the remaining patients' previous diagnostic procedures were performed, mainly head and neck/chest/abdominal CT.

From 53 investigated patients, the primary sites were correctly located in 19 cases (true positive, $36 \%$, Table 2).

The primary tumor could not be identified in 31 (58\%) patients. Twenty- four of these patients were true negative (TN), with no FDG positive findings suggestive of the primary tumor and no proven primary malignancy by further investigations. In the remaining 7 patients, $18 \mathrm{~F}-\mathrm{FDG}$ $\mathrm{PET} / \mathrm{CT}$ did not detect any lesion, but the primary tumors were detected during clinical follow-up/further investigations (Table 3). 
In our study, we had only three false-positive (FP) results (Table 4). One of them was suspected to have lymphoma, because of extensive lymph node involvement, combined with metastatic lesions in other organs, which turn out to be lung carcinoma. In the other case, there was intense FDG uptake in the stomach, suspicious for malignancy, but the endoscopic examination showed no evidence of malignancy. In the last case, we suspected a primary location in the submandibular gland, but it wasn't confirmed as a primary and turned out to be metastatic lymph nodes. A false-positive case is presented in Figure 1.

Patients were divided into four groups depending on the initial location of histologically proven or suspected by conventional imaging modalities LNM.

Group 1: Cervical LNM. A total of 39 patients presented initially with cervical LNM. PET/CT suggested
Table 1. Patients' and disease characteristics.

\begin{tabular}{lc}
\hline Patients' and disease characteristics & Number \\
\hline Male (n) & 34 \\
Female (n) & 19 \\
Age (years) & $35-72$ (median 58) \\
Histology of lymph nodes & 23 \\
$\quad$ squamous cell carcinoma & 9 \\
poorly differentiated carcinoma & 6 \\
undifferentiated carcinoma & 4 \\
adenocarcinoma & 4 \\
without histology & 7 \\
others rarer histology variants (melanoma, NET) & \\
Prior diagnostic work-up & 43 \\
(+) & 10 \\
(-) & \\
\hline
\end{tabular}

Table 2. True-positive results diagnosed by $18 \mathrm{~F}$-fluorodeoxyglucose positron emission tomography/computed tomography.

\begin{tabular}{|c|c|c|c|}
\hline Age and gender & Location of metastases & Histopathologic / radiologic metastases & Primary tumor \\
\hline $1.61, \mathrm{M}$ & Cervical LN & Small-cell blastoma & Nasopharynx \\
\hline 2. $66, \mathrm{~F}$ & Cervical LN & Adenocarcinoma & Lung \\
\hline 3. $44, \mathrm{M}$ & Cervical LN & Squamous cell carcinoma & Oropharynx \\
\hline 4. $62, \mathrm{~F}$ & Axillary LN & Adenocarcinoma & Breast \\
\hline 5. $48, \mathrm{M}$ & Retroperitoneal LN & Papillary mucinous neoplasm & Pancreas \\
\hline 6. $61, \mathrm{M}$ & Cervical LN & Squamous cell carcinoma & Larynx \\
\hline 7. $62, \mathrm{~F}$ & Pelvic LN & Radiologic metastases & Lymphoma \\
\hline 8. $63, \mathrm{~F}$ & Cervical LN & Squamous cell carcinoma & Tonsilla palatina \\
\hline 9. $61, \mathrm{M}$ & Cervical LN & Squamous cell carcinoma & Nasopharynx \\
\hline 10. $67, \mathrm{M}$ & Cervical LN & Squamous cell carcinoma & Lung \\
\hline 11. $59, \mathrm{M}$ & Cervical LN & Squamous cell carcinoma & Tongue \\
\hline 12. $38, \mathrm{M}$ & Cervical LN & Squamous cell carcinoma & Tongue \\
\hline 13. $56, \mathrm{M}$ & Cervical LN & Epithelial carcinoma & Lung \\
\hline 14. $70, \mathrm{M}$ & Cervical LN & Squamous cell carcinoma & Lung \\
\hline 15. $44, \mathrm{M}$ & Cervical LN & Undifferentiated carcinoma & Nasopharynx \\
\hline 16. $38, \mathrm{M}$ & Cervical LN & Squamous cell carcinoma & Maxillary sinus \\
\hline 17. $72, \mathrm{M}$ & Cervical LN & Adenocarcinoma & Prostate \\
\hline 18. $50, \mathrm{M}$ & Axillary LN & Poorly differentiated carcinoma & Lung \\
\hline 19. $60, \mathrm{~F}$ & Axillary LN & Poorly differentiated carcinoma & Breast \\
\hline
\end{tabular}

Abbreviations: LN-Lymph node; M-Male; F-Female; 18F-FDG-18F-fluorodeoxyglucose; PET/CT-Positron emission tomography/computed tomography

Table 3. False-negative results of $18 \mathrm{~F}$-fluorodeoxyglucose positron emission tomography/computed tomography in patients with carcinoma of unknown primary.

\begin{tabular}{|c|c|c|c|}
\hline Age and gender & Location of metastases & Final diagnosis & Pathology of primary tumor \\
\hline $1.65, \mathrm{~F}$ & Cervical LN & Nasopharyngeal cancer & SCC \\
\hline 2. $71, \mathrm{M}$ & Cervical LN & Lymphoma & DLBCL \\
\hline 3. $60, \mathrm{~F}$ & Axillary LN & Breast cancer & Lobular cancer \\
\hline 4. $66, \mathrm{M}$ & Cervical LN & Lung cancer & SCC \\
\hline 5. $67, \mathrm{M}$ & Cervical LN & Parotid tumor & Adenocarcinoma \\
\hline 6. $60, \mathrm{M}$ & Cervical LN & Oropharyngeal cancer & SCC \\
\hline 7. $36, \mathrm{M}$ & Cervical LN & Retroperitoneal sarcoma & Desmoplastic small-round-cell tumor \\
\hline
\end{tabular}

Abbreviations: LN-Lymph node; M-Male; F-Female; Scc-Squamous cell carcinoma; DLBCL-Diffuse large B-cell lymphoma 
Table 4. False-positive results diagnosed by $18 \mathrm{~F}$-fluorodeoxyglucose positron emission tomography/computed tomography.

\begin{tabular}{lccc}
\hline Age and gender & Location of metastases & PET/CT diagnosis & Pathology of lesion \\
\hline 1. $79, \mathrm{~F}$ & Cervical LN & Lymphoma & TET \\
$2.48, \mathrm{~F}$ & Cervical LN & Gastric cancer & Lung cancer \\
3. $50, \mathrm{M}$ & Cervical LN & Submandibular gland & Adenosquamous carcinoma \\
\hline
\end{tabular}

Abbreviations: LN-Lymph node; M-Male, F-Female; CUP-Carcinoma of unknown primary; PET/CT-Positron emission tomography/computed tomography; NET-Neuroendocrine tumors

A

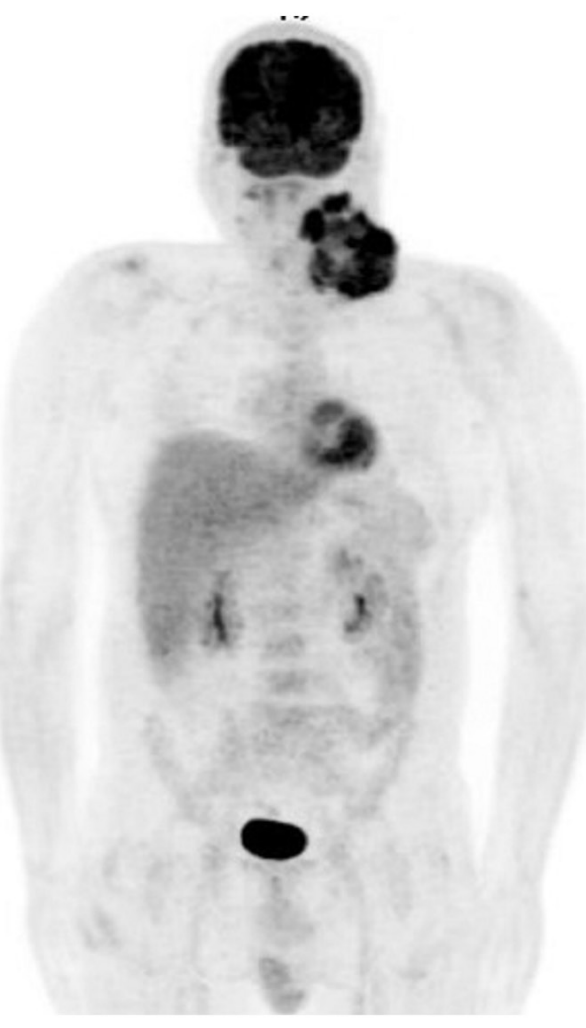

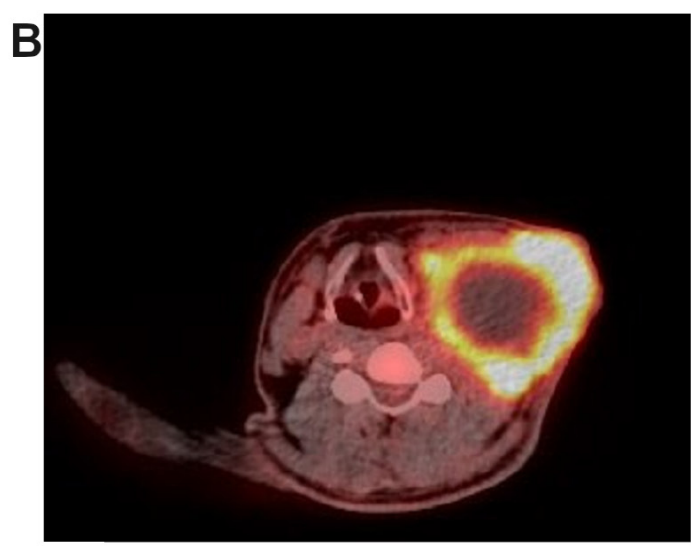

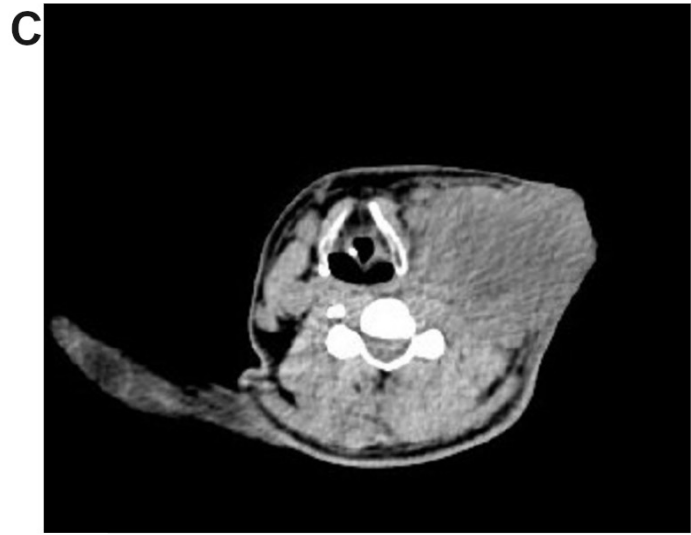

D

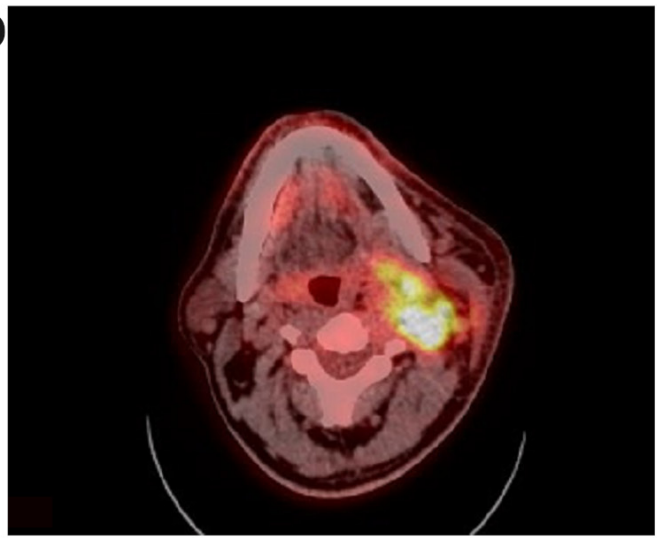

Figure 1. PET/CT in a 50-year-old man with cervical adenopathy. Histology revealed adenocarcinoma. A) Maximum projection intensity image showing a focus of increased FDG uptake in a large tumor mass in the left cervical region. B) Transaxial fused PET/CT section showing increased FDG uptake in the periphery of a formation relative to the center, corresponding to the heterogeneous structure of the lesion in (C) transaxial CT imagechanges consistent with possible central necrosis. D) Transaxial fused PET/CT showing additional FDG avid enlarged left cervical lymph nodes. The patient was suspected to have primary carcinoma of the submandibular gland, but further investigations and follow up revealed extensive lymph node metastasis-FP. 
probable primary in 17 cases (lung [4], nasopharynx [3], tongue [2], larynx [1], tonsilla palatine [1], oropharynx [1], submandibular gland [1], maxillary sinus [1], lymphoma [1], stomach [1], and prostate [1]). All these sites of the indicated primary were found to be true positive (14 out of 39 cases, 36\% true positive), except in 3 patients (submandibular gland [1], lymphoma [1], and stomach [1]), where it was found to be metastatic lymph nodes, lung carcinoma, and inflammation, respectively (false positive). PET/CT could not locate the site of the primary in 22 out of 39 patients with cervical LNM. In 6 of the latter, the primary site was detected by further investigations (nasopharynx [1], lymphoma [1], parotid gland [1], lung [1], oropharynx [1], and retroperitoneal sarcoma [1]), hence were considered false negative. In the remaining 16 patients, despite extensive work-up and follow-up, the site of primary remained unknown (true negative). From this group of patients, we represent an example of a true positive case with LNM in the cervical region which proved to be carcinoma of tonsilla palatine (Figure 2). The sensitivity, specificity, positive predictive value, negative predictive value and accuracy of 18F-FDG PET/CT in this group of patients with cervical lymph node metastases was 70\% (95\% CI 45.7-88.11\%), 84\% (95\% CI 60.4-96.62\%), 82\% (95\% CI $61.3-93.2 \%), 73 \%$ (95\% 57.0-84.26\%), and $77 \%$ (95\% CI 60.6-88.8\%), respectively.

Group 2: Axillary LNM. PET/CT was able to identify correctly the site of the primary in 3 out of 6 patients (50\%), one patient with lung cancer and two patients with breast carcinoma, which were confirmed by a biopsy and proved to be a true positive (Figure 3 ). In the rest 3 patients, PET/ CT was not able to detect the site of the primary. Two of these patients remained with a diagnosis of CUP and in one a breast carcinoma was found during the follow-up.
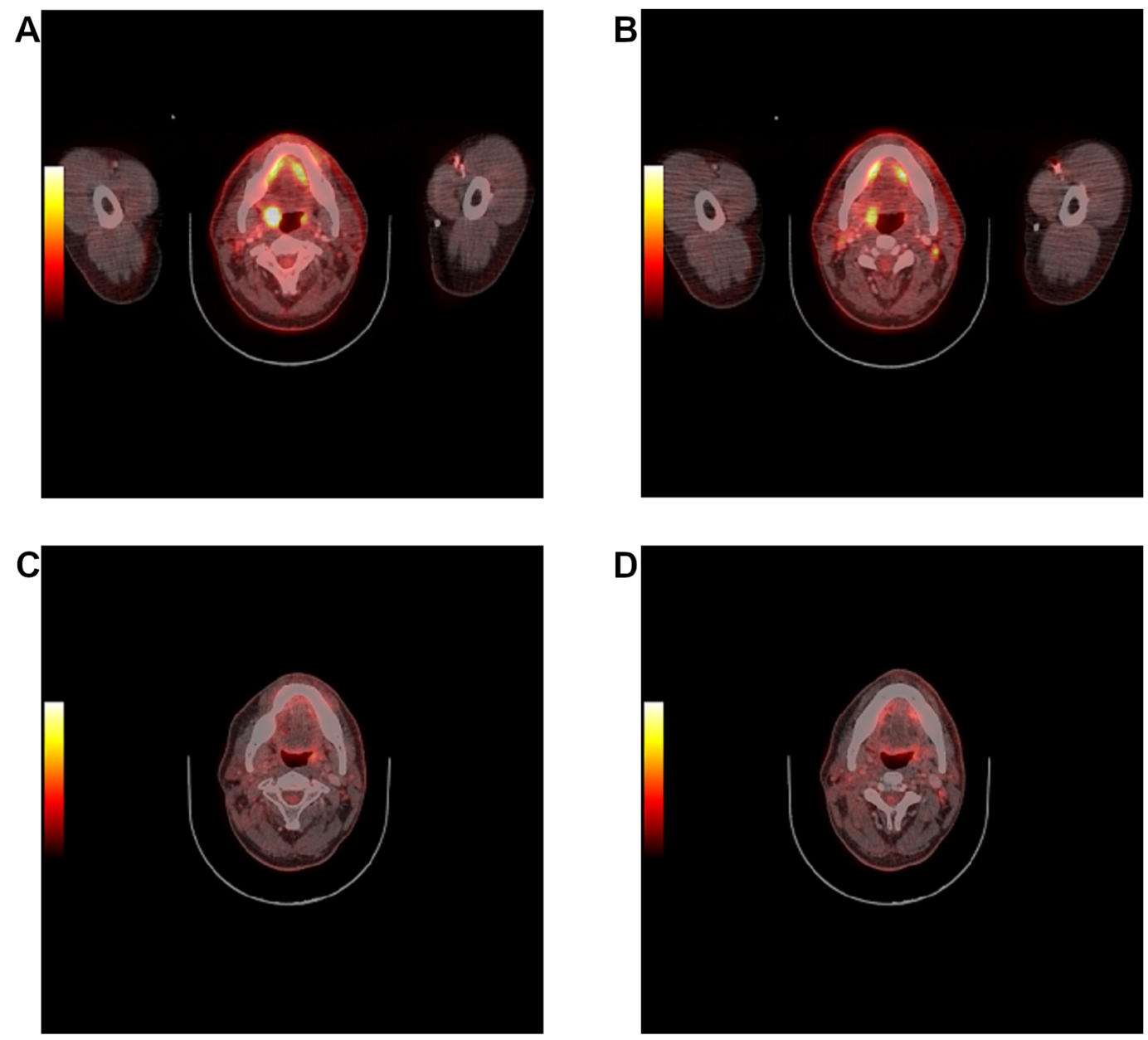

Figure 2. PET/CT in a male patient with cervical adenopathy of squamous origin. Previous diagnostic CT was negative for a potential primary localization. A, B) Transaxial fused PET/CT images showing the focus of asymmetrical increased FDG uptake (SUVmax 10.7) in right tonsil palatine suggestive of the probable primary tumor and moderate FDG uptake (SUVmax 3.8) in cervical lymph nodes of level II. Tonsillectomy with lymph node dissection was performed (histology was negative for metastatic involvement of suspected FDG avid lymph nodes!). The patient received chemo- and radiotherapy. The restaging PET/CT (C, D) showed no evidence of recurrence and dissemination. 
Group 3: Mediastinal LNM. PET/CT was totally negative without evidence for primary location, neither any FDG-avid metastatic foci. The patients were followed up and proved to be true negative.

Group 4: Abdominal and pelvic LNM. The possible primary location was suggested in 2 of 6 cases (33\%) one patient with pancreatic cancer and one with lymphoma (true positive). The site of the primary tumor remained unknown in the rest four patients, even after extensive workup and follow-up (true negative).

In addition to the previously known metastases, 18F-FDG PET/CT identified additional distant metastatic foci in 21 of all patients (40\%) the most frequent localization in other lymph nodes groups, lung, bones, and liver, thus changing the therapeutic management of a significant number of cases (Figure 4).

The overall sensitivity, specificity and accuracy rates of 18F-FDG PET/CT in the detection of a primary tumor in patients with LNM were identified as 73\% (95\% CI 52.2-88.4\%), 89\% (95\% CI: 70.8-97.6\%), and 81\% (95\% CI: 68.0-90.5\%), respectively. Receiver operating characteristic
(ROC) curves were constructed, and the area under the curve (AUC) was calculated. The ROC analysis showed overall good accuracy of PET/CT in the identification of suggestive primary tumor, with an area under the ROC curve of 0.799 (95\% CI: 0.66-0.89, $\mathrm{p}=0.001$, Figure 5).

In our study, only ten of 53 patients (19\%) were without any FDG-avid primary locations or other FDG positive findings suggestive for distant metastases. Eight of them were considered as $\mathrm{TN}$, while in two cases a carcinoma of the oropharynx and breast cancer were found by further investigations and they were considered a false-negative. In the rest 43 patients (81\%), the PET/CT result guided the subsequent therapeutic management according to the extent of the disease burden-systemic, combined or local. As a result of the 18F-FDG PET/CT scan, the treatment plans of 26 patients (49\%) required modification. Avoiding unnecessary surgical or systemic procedures was achieved in 15/26 (23\%) and $8 / 26(31 \%)$, respectively. The average survival rate after $\mathrm{PET} / \mathrm{CT}$ exam in our study is 257.2 days.

In $22 / 43$ of the cases where the two imaging procedures were available, the direct comparison between PET/CT and

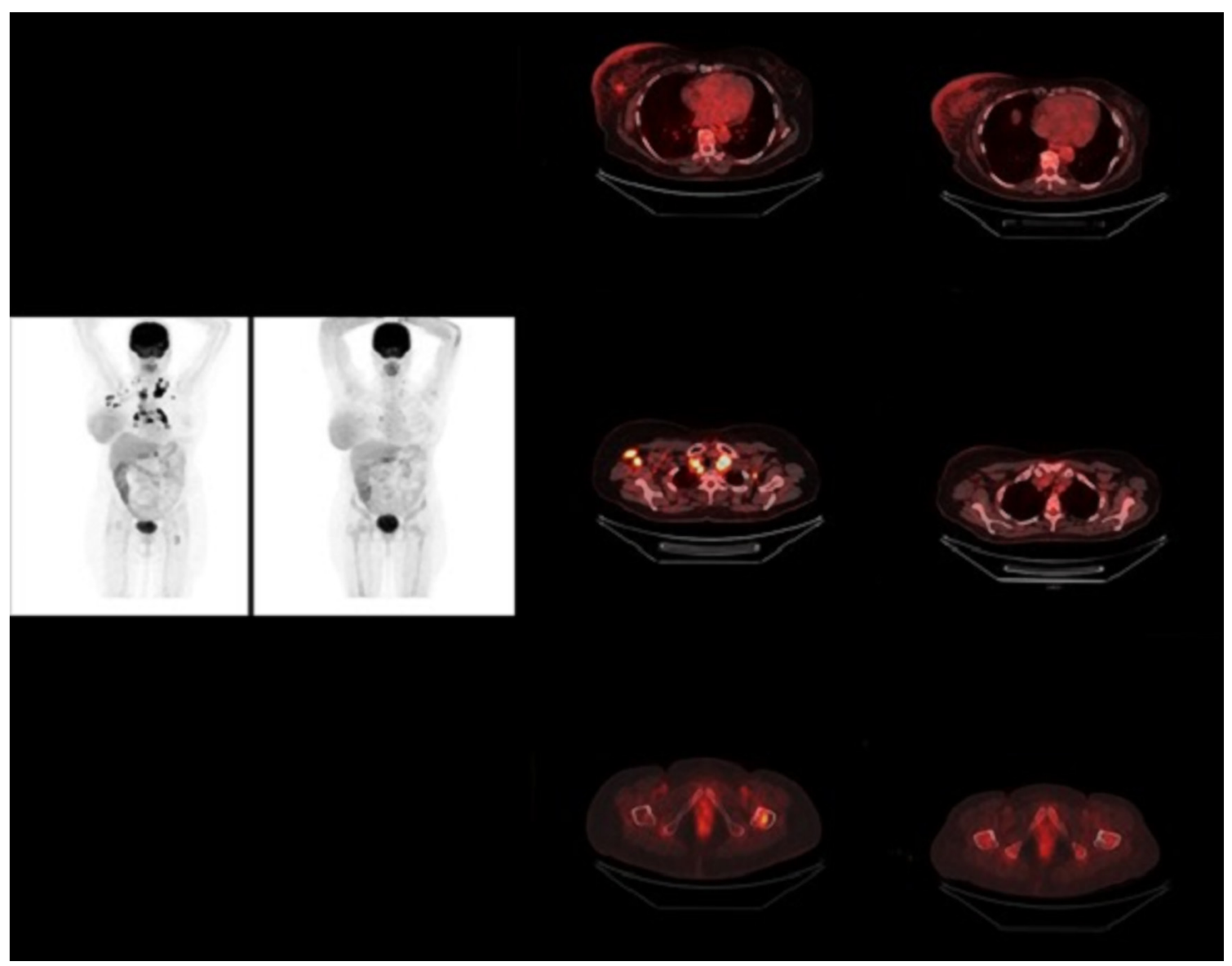

Figure 3. PET/CT in a 68-years-old woman with histology of undifferentiated carcinoma from cervical lymph node metastasis. A staging PET/CT was performed (left side column)-proven right breast carcinoma and dissemination in cervical, axillar, mediastinal, and abdominal lymph nodes and bone lesion. After several cycles of chemotherapy, a follow-up PET/CT showed a significant morphological and metabolically response (right side column). 
CT revealed $51 \%$ coincidence of the final result. In the rest 21 of 43 (49\%) of the cases, the CT result did not confirm the PET finding. The CT showed no morphological changes or was unable to detect the primary/metastatic foci.

\section{Discussion}

It is well known that CT and MRI are still leading imaging methods of choice in clinical practice in patients with CUPs. They are primarily based on anatomical evidence of the increase in size, asymmetry, anatomical distortion, and contrast enhancement. In contrast, molecular imaging as $18 \mathrm{~F}-\mathrm{FDG} \mathrm{PET} / \mathrm{CT}$ is able to detect very early changes in the tumor on a metabolic level. Gutzeit et al. [12] have shown that CT alone indicated a primary tumor in only 8 of 45 patients (18\%), while $18 \mathrm{~F}-\mathrm{FDG}$ PET/CT detected the primary site in 15 of 45 patients (33\%). These data correspond well with our results. In almost one-half of the cases where the two imaging modalities were available, the CT result did not confirm the PET finding. Thus, Kwee et al. [13] emphasized that if FDG $\mathrm{PET} / \mathrm{CT}$ fails to detect a primary tumor, other diagnostic procedures are also likely to fail, so $18 \mathrm{~F}-\mathrm{FDG} \mathrm{PET} / \mathrm{CT}$ should be used as a first-line imaging modality in these specific patients' population. According to the ESMO Guideline [3], whole-body 18F-FDG-PET/CT may contribute to the management of patients with lymph node involvement of CUP and those with a single CUP metastasis. However, the clinical practice shows a lack of systematic approaches to these patients with regard to their management and diagnosis based on available clinical information, histology, and the most likely area of the primary tumor site. For example, in our study, 18F-FDG PET/CT was the first imaging method in 10 of 53 patients and was able to detect correctly the primary in more than one-half of them, reducing the need for further investigation.

Previous studies had indicated that the most frequently identified primaries in CUP by $18 \mathrm{~F}-\mathrm{FDG}$ PET/CT were lungs or head and neck carcinoma $[14,15]$, as the results of our study. 18F-FDG PET/CT has proven its usefulness in localizing a primary tumor in the head-neck area compared to contrast-enhanced CT and MRI and could be recommended as an early diagnostic modality in the workup of these patients $[16,17]$. Compared to the other localization of LNM, our data suggests the highest sensitivity and specificity of FDG PET/CT in the group with cervical LNM-70\% and $84 \%$, respectively. This result corresponds well with a study of Roh et al. [18], reporting that the sensitivity rate of $18 \mathrm{~F}-\mathrm{FDG}$ PET/CT (87.5\%) was significantly higher than CT $(43.7 \%)$ for primary tumor detection in patients with cervical LNM.

We could not detect with 18 F-FDG PET/CT the primary tumor in 31 (58\%) of the patients, seven of these were considered as FN. This result could be explained by the relatively unspecific characteristics of 18F-FDG PET/CT as a tumor imaging technique. A recent meta-analysis showed that the oropharynx and the lungs are the two most common

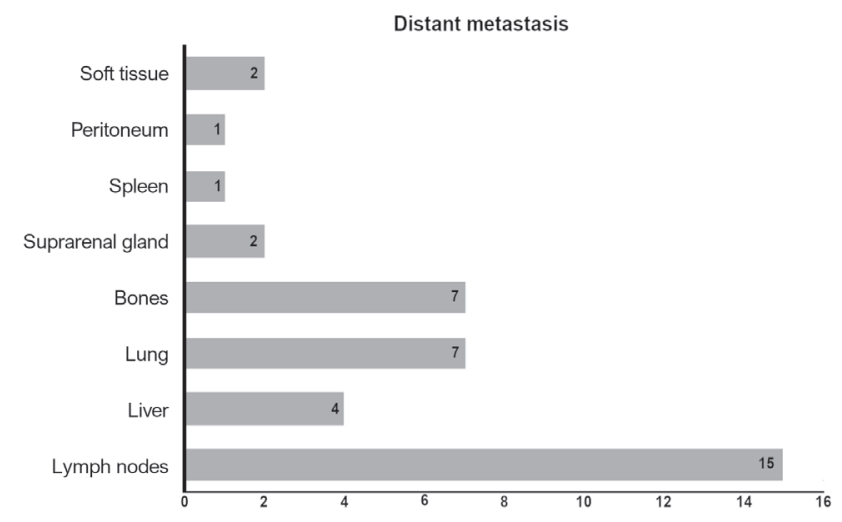

Figure 4. Distribution of additional metastatic disease detected by $18 \mathrm{~F}-$ FDG PET/CT.

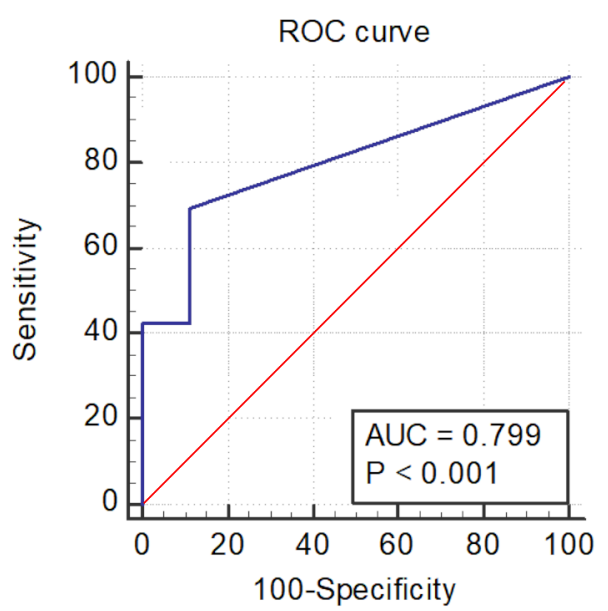

Figure 5. ROC curve and corresponding AUC statistics for diagnostic efficiency of PET/CT in suggestive primary neoplasm. Overall accuracy was good, with area under ROC curve of 0.799 (95\% CI: 0.66-0.89, p=0.001).

locations of false-positive 18F-FDG PET/CT results [19]. In the setting of head and neck cancer, the limitation of PET/ CT is due to the resolution of FDG-PET, limited to approximately $5 \mathrm{~mm}$. Small or superficial lesions may be undetected. The basal uptake of FDG in the normal lymphoid tissues of the Waldeyer ring and salivary glands would further obscure the detection of small and superficial lesions [20]. Additionally, when considering possible breast carcinoma there are a number of sources for false-negative results on $18 \mathrm{~F}-\mathrm{FDG}$ $\mathrm{PET} / \mathrm{CT}$ as low-grade and well-differentiated tumors, some histological tumor type (tubular carcinoma, lobular carcinoma, in situ carcinoma). Mammography and/or ultrasonography are highly recommended [1]. According to the literature, MRI of the breast should be used in patients with negative clinical examination, mammography, or ultrasound and is able to detect between $50-86 \%$ of the primary occult breast carcinomas [21, 22].

Special attention should be paid to the clinical scope of axillary LNM from unknown primary origin, given the high 
rate of false-negative results with respect to local status. Thus, we also support the thesis and conclusions from various studies that the use of PET/CT in this clinical setting has a complementary role for the overall assessment of distant metastatic foci rather than being the leading imaging technique for identification of local status.

One of the main advantages of 18F-FDG PET/CT is the opportunity to detect distant lesions and perform a complete staging in one procedure. Determination of the exact extent of metastatic disease has important implications for clinical management, particularly in avoiding unnecessary surgical or systemic procedures. Approximately one-third of all patients (29.4 to $33.8 \%$ ) changed their treatment strategy, guided by F-18 FDG PET/CT according to literature data [23-25]. Similar results are reported in another study, confirming that F-18 FDG PET/CT led to treatment modification in $29 \%$ of all patients and in particular $32.2 \%$ of head and neck metastases patients [26]. We showed additional solid organ and lymphatic metastases in $40 \%$ of the studied patients on PET/ CT imaging, leading to treatment modification in almost half of the cases. Indeed, its ability to rule out additional metastatic sites may particularly be of interest in patients with CUP who present with lymph node metastatic disease only, because $M$ stage, which has important therapeutic and prognostic consequences, is still unknown in these patients. Also, the detection of additional lymph node involvement based upon the FDG PET/CT study might help in modifying and planning the radiotherapy field appropriately, which is the pivotal modality of therapy in these patients. Furthermore, a baseline FDG PET/CT may also play a valuable role in treatment monitoring following therapeutic intervention. Compared to other diagnostic procedures that are often used in patients with CUP (e.g., CT alone and endoscopic procedures), FDG PET/CT is both noninvasive and a very sensitive tomographic whole-body imaging modality, allowing for the detection of a primary tumor and complete tumor staging in a single examination. Pulmonary carcinoma, on the other hand, is the second most common primary localization in the clinical scenario of cervical metastases from an occult primary. In regard to non-small cell lung cancer (NSCLC), PET/CT scans have proven to be statistically more accurate than any other method, as far as tumor staging is concerned. As a result of the exact correlation between anatomy and functional disorder, focal chest wall infiltration, mediastinal invasion, and differentiation of tumor from atelectasis have largely improved with PET/CT. Unexpected extrathoracic soft tissue or skeletal metastases also may be revealed by PET/CT in cases where other imaging methods failed to demonstrate distant metastasis. The extent of tumor spread is the primary factor that determines whether the patient will undergo surgery or will be offered a non-surgical treatment. In the staging of NSCLC PET/CT may result in an alteration of treatment management in up to 30 percent of patients.

However, it seems that the lack of homogenous cohort large scale prospective studies validating the use of F-18
FDG PET/CT and its cost-effectiveness in CUP patients has prevented the routine application of this imaging modality as a part of standard work in CUP $[13,27,28]$. Also, the 18F-FDG PET/CT procedure is associated with significantly higher costs, a longer examination time, and much more limited availability at all hospitals, which may further limit its use as an initial imaging modality.

Nevertheless, we believe that early application of $18 \mathrm{~F}-\mathrm{FDG}$ $\mathrm{PET} / \mathrm{CT}$ in the staging process may save time and unnecessary investigations with proved benefits in certain patterns, in particular in those with cervical lymph nodes metastasis.

Our study had a few limitations - small sample size, retrospective nature, a heterogeneous cohort of patients with CUP syndrome, and previously conducted therapies in some of the patients before the PET/CT exam, which could influence the detection rate of possible primary locations. Based on our observations and data from other literature sources, we strongly recommend histological confirmation of metastatic carcinoma of unknown primary due to the unspecific nature of PET/CT as an oncology imaging technique and the low or missing 18F-FDG avidity sensitivity to certain tumor entities, such as renal cell carcinoma, hepatocellular carcinomas, mucinous carcinomas or low-grade lymphomas. Histological confirmation of suspected primary location is also recommended in order to avoid false-positive cases, which could mimic the malignant process.

Despite the small number of our patients and heterogeneity in diagnostic workups, we believe that FDG PET/CT is a more sensitive and selective procedure for detecting unknown primary tumors, especially in the clinical setting of cervical LNM. The main advantages of this hybrid modality in the pre-treatment phase of CUPs are the localization of probable primary sites and guiding biopsy, cervical lymph node staging, detection of metastases, and finding of synchronous second primary malignancies. We support the idea that the use of 18F-FDG PET/CT should be encouraged earlier in CUP patients whenever possible and especially if executed as positron emission tomography-contrast enhanced computed tomography (PET-CECT), which could provide also sufficient anatomic information for treatment planning. Therefore, perhaps FDG PET/CT should be used as a first-line imaging modality in all patients with the metastatic disease in lymph nodes rather than using it after other diagnostic procedures have failed to identify a primary tumor. This possible beneficial shift in the diagnostic workup of the patients with metastatic disease requires further investigation.

In conclusion, whole-body $18 \mathrm{~F}-\mathrm{FDG}$ PET/CT is an effective method for detecting the primary tumor in patients with CUP. We consider that it can play a key role in both the diagnostic workup and the selection of curative or palliative treatment in a patient with cervical lymph node metastases and perhaps should be used as a first-line imaging modality in this setting. Further studies are needed to determine its exact position in the diagnostic workup and its potential role in patient outcome. 


\section{References}

[1] PAVLIDIS N, BRIASOULIS E, HAINSWORTH J, GRECO FA. Diagnostic and therapeutic management of cancer of an unknown primary. Eur J Cancer 2003; 39: 1990-2005. https://doi.org/10.1016/s0959-8049(03)00547-1

[2] BRIASOULIS E, PAVLIDIS N. Cancer of unknown primary origin. Oncologist 1997; 2: 142-152.

[3] FIZAZI K, GRECO FA, PAVLIDIS N, DAUGAARD G, OIEN K et al. Cancers of unknown primary site: ESMO Clinical Practice Guidelines for diagnosis, treatment and followup. Ann Oncol 2015; 26: v133-138. https://doi.org/10.1093/ annonc/mdv305

[4] PEJČIĆ I, VRBIĆ S, TODOROVIĆ M, PETKOVIĆ I, BALIĆ M et al. Cancer of Unknown Primary Site SyndromeCUP Syndrome - Diagnostic and Therapeutical Dilemmas. Acta Facultatis Medicae Naissensis 2014; 31: 87-94. https:// doi.org/10.2478/afmnai-2014-0010

[5] COLLADO MARTÍN R, GARCÍA PALOMO A, DE LA CRUZ MERINO L, BORREGA GARCÍA P, BARÓN DUARTE FJ. Clinical guideline SEOM: cancer of unknown primary site. Clin Transl Oncol 2014; 16: 1091-1097. https:// doi.org/10.1007/s12094-014-1244-0

[6] PAVLIDIS N, KHALED H, GAAFAR R. A mini review on cancer of unknown primary site: A clinical puzzle for the oncologists. J Adv Res 2015; 6: 375-382. https://doi. org/10.1016/j.jare.2014.11.007

[7] LE CHEVALIER T, CVITKOVIC E, CAILLE P, HARVEY J, CONTESSO G et al. Early metastatic cancer of unknown primary origin at presentation. A clinical study of 302 consecutive autopsied patients. Arch Intern Med 1988; 148: 2035-2039.

[8] FERRER RJ, WAHL RL. Carcinoma of unknown primary, including paraneoplastic neurological syndromes. In: Wahl RL, editor. Principles and Practice of PET and PET/CT. 2nd ed. Philadelphia, PA: Lippincott Williams and Wilkins; 2009: 438-442.

[9] RUSTHOVEN KE, KOSHY M, PAULINO AC. The role of fluorodeoxyglucose positron emission tomography in cervical lymph node metastases from an unknown primary tumor. Cancer 2004; 101: 2641-2649. https://doi.org/10.1002/ cncr.20687

[10] SÈVE P, BILLOTEY C, BROUSSOLLE C, DUMONTET C, MACKEY JR. The role of 2-deoxy-2-[F-18] fluoro-D-glucose positron emission tomography in disseminated carcinoma of unknown primary site. Cancer 2007; 109: 292-299. https:// doi.org/10.1002/cncr.22410

[11] JAIN A, SRIVASTAVA MK, PAWASKAR AS, SHELLEY S, ELANGOVAN I et al. Contrast-enhanced [18F] fluorodeoxyglucose-positron emission tomography-computed tomography as an initial imaging modality in patients presenting with metastatic malignancy of undefined primary origin. Indian J Nucl Med 2015; 30: 213-220. https://doi. org/10.4103/0972-3919.158529

[12] GUTZEIT A, ANTOCH G, KÜHL H, EGELHOF T, FISCHER $M$ et al. Unknown primary tumors: detection with dual-modality PET/CT--initial experience. Radiology 2005; 234: 227-234. https://doi.org/10.1148/radiol.2341031554
[13] KWEE TC, BASU S, CHENG G, ALAVI A. FDG PET/CT in carcinoma of unknown primary. Eur J Nucl Med Mol Imaging 2010; 37: 635-644. https://doi.org/10.1007/s00259-0091295-6

[14] ELBOGA U, KERVANCIOGLU S, SAHIN E, BASIBUYUK $\mathrm{M}$, CELEN YZ et al. Utility of F-18 fluorodeoxyglucose positron emission tomography/computed in carcinoma of unknown primary. Int J Clin Exp Pathol 2014; 7: 8941-8946.

[15] GÜL SS. The Diagnostic Contribution of (18)F-FDG PET/ CT Scan in Cancer of Unknown Primary. Cumhuriyet Med J 2018; 40: 238-245. https://doi.org/10.7197/223.vi.412273

[16] OLDAN JD, YOO DS, LEE WT, HOANG JK. PET/CT for Head and Neck Squamous Cell Cancer-Uses and Updates for Radiologists. Curr Radiol Rep 2014; 2. https://doi. org/10.1007/s40134-014-0047-y

[17] PODOLOFF DA. PET/CT and Occult Primary Tumors. J Natl Compr Canc Netw 2009; 7: 239-244. https://doi. org/10.6004/jnccn.2009.0019

[18] ROH JL, KIM JS, LEE JH, CHO KJ, CHOI SH et al. Utility of combined (18)F-fluorodeoxyglucose-positron emission tomography and computed tomography in patients with cervical metastases from unknown primary tumors. Oral Oncol 2009; 45: 218-224. https://doi.org/10.1016/j.oraloncology.2008.05.010

[19] KWEE TC, KWEE RM. Combined FDG-PET/CT for the detection of unknown primary tumors: Systematic review and meta-analysis. Eur Radiol 2009; 19: 731-744. https://doi. org/10.1007/s00330-008-1194-4

[20] GUARISCHI A, KEANE TJ, ELHAKIM T. Metastatic inguinal nodes from an unknown primary neoplasm. A review of 56 cases. Cancer 1987; 59: 572-577. https:// doi.org/10.1002/1097-0142(19870201)59:3<572::aidcncr2820590336>3.0.c0;2-3

[21] BUCHANAN CL, MORRIS EA, DORN PL, BORGEN PI, VAN ZEE KJ. Utility of breast magnetic resonance imaging in patients with occult primary breast cancer. Ann Surg Oncol 2005; 12: 1045-1053. https://doi.org/10.1245/ ASO.2005.03.520

[22] OREL SG, WEINSTEIN SP, SCHNALL MD, REYNOLDS CA, SCHUCHTER LM et al. Breast MR imaging in patients with axillary node metastases and unknown primary malignancy. Radiology 1999; 212: 543-549. https://doi. org/10.1148/radiology.212.2.r99au40543

[23] WANG G, WU Y, ZHANG W, LI J, WU P et al. Clinical value of whole-body F-18 fluorodeoxyglucose positron emission tomography/computed tomography in patients with carcinoma of unknown primary. J Med Imaging Radiat Oncol 2013; 57: 65-71. https://doi.org/10.1111/j.17549485.2012.02441.x

[24] ELBOGA U, KERVANCIOGLU S, SAHIN E, BASIBUYUK M, CELEN YZ et al. Utility of F-18 fluorodeoxyglucose positron emission tomography/computed in carcinoma of unknown primary. Int J Clin Exp Pathol 2014; 7: 8941-8946.

[25] HU M, ZHAO W, ZHANG PL, JU GF, FU Z et al. Clinical applications of $18 \mathrm{~F}$-fluorodeoxyglucose positron emission tomography/computed tomography in carcinoma of unknown primary. Chin Med J 2011; 124: 1010-1014. 
[26] YU X, LI X, SONG X, DAI D, ZHU L et al. Advantages and Disadvantages of F-18 Fluorodeoxyglucose Positron Emission Tomography/Computed Tomography in Carcinoma of Unknown Primary. Oncol Lett 2016; 12: 3785-3792. https:// doi.org/10.3892/ol.2016.5203
[27] SAIF MW, TZANNOU I, MAKRILIA N, SYRIGOS K. Role and Cost Effectiveness of PET/CT in Management of Patients with Cancer. Yale J Biol Med 2010; 83: 53-65.

[28] BAKHSHAYESHKARAM M, GHOBADI M, HASSANZAD M, DOROUDINIA A, JAMAATI HR et al. Diagnostic Performance of F-18 FDG PET/CT in Patients with Cancer of Unknown Primary: Additional Benefit over CT-Based Conventional Work up. Novel Biomed 2016; 4: 5-12. https:// doi.org/10.22037/nbm.v4i1.10496 